\title{
PRIMER REGISTRO DE Digitonthophagus gazella (Fabricius, 1787) PARA EL DEPARTAMENTO DE SUCRE, COLOMBIA
}

\author{
FIRST REPORT OF Digitonthophagus gazella (Fabricius, 1787) FOR THE \\ DEPARTMENT OF SUCRE, COLOMBIA
}

\begin{abstract}
NAVARRO, I. LUIS, ${ }^{1^{\star}}$ Est, Biología ; ROMAN, A. KENNYA. ${ }^{1}$ Est Biología; GOMEZ, F. HERNANDO. ${ }^{2}$ Biólogo-Botánico; PEREZ, H. ANTONIO ${ }^{2}$.M.Sc.

${ }^{1}$ Facultad de Educación y Ciencias, Departamento de Biología, Universidad de Sucre, Sincelejo Colombia, ${ }^{2}$ Docentes, Facultad de Educación y Ciencias, Departamento de Biología, Universidad de Sucre, Colombia *Correspondencia: lhodwig124@hotmail.com
\end{abstract}

\section{Resumen}

Se posee poca información de la fauna de escarabajos coprófagos del departamento de Sucre, por lo que se llevaron a cabo una serie de muestreos para estimar la diversidad de estos. Se realizaron 4 colectas entre los meses de junio y agosto de 2008, en un área empleada para la ganadería y la agricultura ubicada en la serranía de Coraza, Colosó - Sucre. Se utilizaron dos tipos de trampas: de caída y de interceptación de vuelo, complementado con captura manual. Se capturaron 44 individuos de Digitonthophagus gazella, este constituye el primer reporte para el departamento de Sucre (Colombia).

Palabras clave: Escarabajos coprófagos; ganadería, agricultura; serranía de Coraza, Digitonthophagus gazella

\section{Abstract}

Lack knowledge of dung beetles of Sucre's department is possessed, so they were carried out a series of samplings to estimate the diversity of these. They were 
carried out 4 collections between june and august 2008, in an area used for the cattle-raising and agriculture located in the Serranía de Coraza, Colosó - Sucre. Using two types of traps: pitfall traps and flight interception, supplemented with manual capture. 44 individuals of Digitonthophagus gazella were captured. This constitutes the first report for the department of Sucre (Colombia).

Key words: dung beetles; cattle-raising, agriculture; serranía de Coraza, Digitonthophagus gazella

\section{Introducción}

Digitonthophagus gazella es una especie afro-asiática, que fue incluida, inicialmente, en el género Onthophagus Latreille 1802, subgénero Digitonthophagus Balthasar 1959, posteriormente en 1981 fue elevada a la categoría de género por Zunino (KOHLMANN, 1994; NORIEGA, 2002). Su presencia, distribución y migración se ha documentado ampliamente, debido a que ha sido introducida en regiones tropicales, subtropicales, áridas y semiáridas del mundo con el fin de contribuir con el control del exceso de excrementos producidos por el aumento de la ganadería (KOHLMANN, 1994).

Esta especie posee una gran capacidad de dispersión, característica que se comprueba al examinar la distribución en el continente americano (RIVERA y WOLFF, 2007). En 1972 fue liberada en Texas (E.E.U.U) como parte de un programa de control de excrementos de ganado vacuno y posteriormente migró hacia otros estados del país (FINCHER et al., 1983; KOHLMANN, 1991). Subsecuentemente, ha llegado a establecerse en otras regiones del continente americano como México y en la península de Yucatán (RIVERA-CERVANTES y GARCIA-REAL, 1991; MONTES DE OCA y HALFFTER, 1997; MORALES et al., 2004), Guatemala (KOHLMANN, 1994), Nicaragua (NORIEGA, 2002), Chile (NORIEGA, 2002), Uruguay (KOHLMANN, 1994) y de manera no oficial en 
Venezuela (NORIEGA et al., 2006). En 1989 fue exportado de Estados Unidos hacia Brasil (BIANCHIN et al., 1998).

En Colombia se registró la presencia de esta especie en territorios insulares (San Andrés) en 1995 (NORIEGA, 2002). En territorios continentales entre 2004 y 2006 en localidades de los departamentos de Atlántico, Bolívar, Casanare, Cesar, La Guajira, Magdalena y Vichada (NORIEGA et al., 2006; RIVERA y WOLFF, 2007).

\section{Materiales y métodos}

Se recolectaron muestras en un área de uso agrícola y ganadero en la serranía de Coraza, municipio de Colosó - Sucre dentro del enclave azonal (Distrito Montes de María y Piojó) perteneciente al Cinturón Árido Pericaribeño (Colombia) según HERNÁNDEZ et al. (1992). Los muestreos corresponden al desarrollo del proyecto "Comparación de la comunidad de escarabajos coprófagos (Coleoptera: Scarabaeidae) en un ecosistema ganadero y en un relicto de bosque seco tropical (Bs - T) del departamento de Sucre".

Para la captura se emplearon trampas pitfall cebadas con excremento humano (TCEH) y bovino (TCEB), trampas de interceptación de vuelo (TIV) y se complementó con búsqueda manual (CM) en las bostas del ganado vacuno. El material colectado se transportó en recipientes plásticos de 2 Oz., limpiado y separado en el laboratorio. Los muestreos se realizaron entre los meses de junio y agosto de 2008, en este periodo se reportó una precipitación promedio mensual de $223.4,218.3$ y $136.1 \mathrm{~mm}$, respectivamente.

Material colectado: se capturaron 44 individuos de los cuales 16 correspondieron a hembras ( + ) y 28 machos $(\AA)$, con una longitud promedio del cuerpo de 10.9 y $11.2 \mathrm{~mm}$, respectivamente. Colectándose 1 individuo en junio, 41 en julio y 2 en agosto. Cuatro de estos ejemplares se depositaron en el Museo Entomológico 
"Francisco Luis Gallego" ubicado en la Universidad Nacional de Colombia sede Medellín [MEFLG].

Material examinado: COLOMBIA. Sucre. 2 + y y 2 , Municipio de Colosó, serranía de Coraza, (9⒉ 29.347 N y 752․ 22.668 W), 220 m, 10 - VII - 2008, NAVARRO y ROMAN, leg. Det. F. ESCOBAR (Diciembre 2008). [MEFLG]

\section{Agradecimientos}

Al Dr. Federico Escobar Sarria por su asesoría y por la determinación taxonómica de los especímenes colectados. Al Museo Entomológico "Francisco Luis Gallego" por permitirnos utilizar sus instalaciones para la identificación taxonómica de los especímenes y su invaluable colaboración. Al Laboratorio de Entomología de la Universidad de Sucre y al Dr. Javier Beltrán, director del Grupo de Investigación de Biotecnología Vegetal. A la comunidad de la Vereda El Paraíso № 1, Colosó, Sucre, y muy especialmente a la familia Mendivil, por su apoyo.

\section{Referencias}

BIANCHIN, I.; ALVES, R.G.O.; KOLLER, W. 1998. Efeito de carrapaciticidas/inseticidas "Pour-on" sobre adultos do besouros coprófago afriacano Onthophagus gazella Fabr. (Coleoptera: Scarabaeidae). An. Soc. Entomol. Brasil 27:275 - 279

FINCHER, G.T.; STEWART, T.B.; HUNTER III, J.S. 1983. The 1981 distribution of Onthophagus gazella Fabricius from releases in Texas and Onthophagus taurus Schreber from an unknown release in Florida (Coleoptera: Scarabaeidae). Coleopterists bulletin 37:159-163

HERNANDEZ, J.; HURTADO, A.; ORTIZ, R.; WALSCHBURGER, T. 1992. Unidades biogeográficas de Colombia. Págs. 105 - 151 en: Halffter, G. (ed) La diversidad biológica de Iberoamérica. Acta Zoológica Mexicana. Volumen especial

KOHLMANN, B. 1991. Dung beetles in subtropical North America Págs. 116 - 132 en: Hanski. I; Cambefort, Y. (eds), Dung beetles ecology. Princeton University Press, New Jersey 
KOHLMANN, B. 1994. A preliminary study of the invasion and dispersal of Digitonthophagus gazella (Fabricius, 1787) in Mexico (Coleoptera: Scarabaeidae: Scarabaeinae). Acta Zoologica Mexicana (n.s) 61:35 - 42

MONTES DE OCA, E.; HALFFTER, G. 1997. Invasion of Mexico by two dung beetles previously introduced into the United States. Studies on Neotropical Fauna and Environment 33:37 - 45

MORALES, C.; RUIZ-NAJERA, R.; DELGADO, L. 2004. Primer registro de Euoniticellus intermedius (Rieche, 1849) y datos nuevos de distribución de Digitonthophagus gazella (Fabricius, 1787) (Coleoptera: Scarabaeidae) e Hybosorus illigery Rieche, 1853 (Coleoptera: Hybosoridae) para el Estado de Chiapas. Dugesiana 11(2):21- 23

NORIEGA, J.A. 2002. First report of the presence of the genus Digitonthophagus (Coleoptera: Scarabaeidae) in Colombia. Caldasia 24(1):213 - 215

NORIEGA, J.A.; SOLIS, C.; QUINTERO, I.; PEREZ, L.G.; GARCIA, H.G.; OSPINO, D.H. 2006. Registro continental de Digitonthophagus gazella (Coleoptera: Scarabaeidae) en Colombia. Caldasia 28(2):379 - 381

RIVERA-CERVANTES, L.E.; GARCIA-REAL, E.1991. New locality records for Onthophagus gazella Fabricius (Coleoptera: Scarabaeidae) in Jalisco, Mexico. Coleopterists Bulletin 45:370

RIVERA, C.; WOLFF, M. 2007. Digitonthophagus gazella (Coleoptera: Scarabaeidae): Distribución en América y dos nuevos registros para Colombia. Revista Colombiana de Entomología 33 (2):190 - 192 